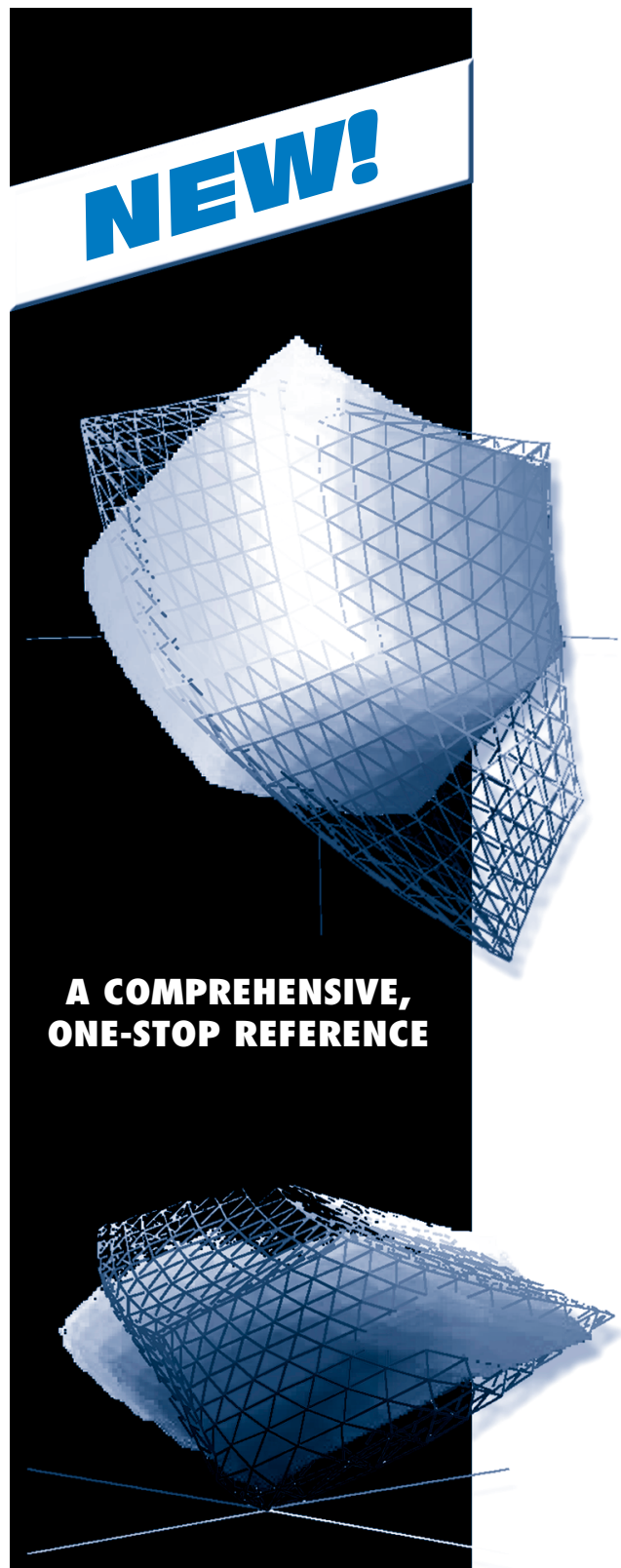

Color your world - Digitally!

\title{
Digital Color Imaging Handbook
}

\author{
Gaurav Sharma \\ Xerox Corporation, Webster, New York, USA
}

A volume in the Electrical Engineering \& Applied Signal Processing series Series Editor: Alexander Poularikis

University of Alabama, Huntsville, USA

\section{A broad-based, authoritative view of color imaging science, algorithms, and technology.}

Digital technology now enables unparalleled functionality and flexibility in the capture, processing, exchange, and output of color images. But harnessing its potential requires knowledge of color science, systems, processing algorithms, and device characteristics-topics drawn from a broad range of disciplines. One can acquire the requisite background with an armload of physics, chem-

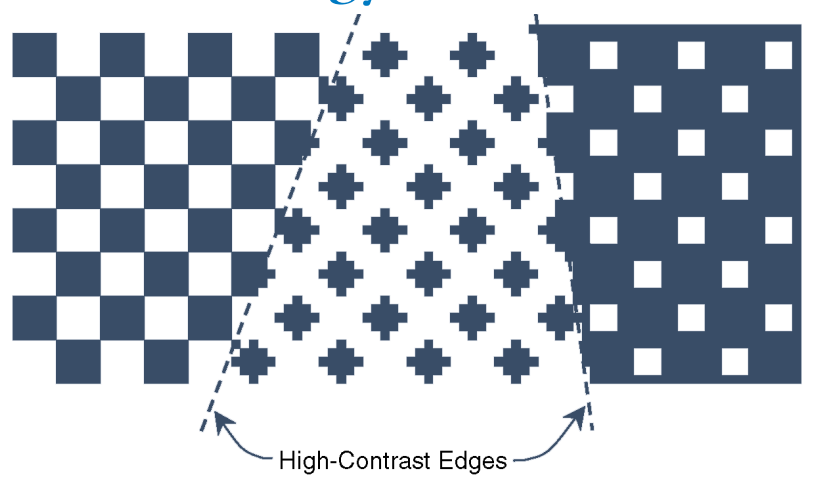
istry, engineering, computer science, and mathematics books and journals - or one can find it here, in the Digital Color Imaging Handbook.

Unprecedented in scope, this handbook presents, in a single concise and authoritative publication, the elements of these diverse areas relevant to digital color imaging. The first three chapters cover the basics of color vision, perception, and physics that underpin digital color imaging. The remainder of the text presents the technology of color imaging with chapters on color management, device color characterization, digital halftoning,

\section{WORLD RENOWNED CONTRIBUTORS}

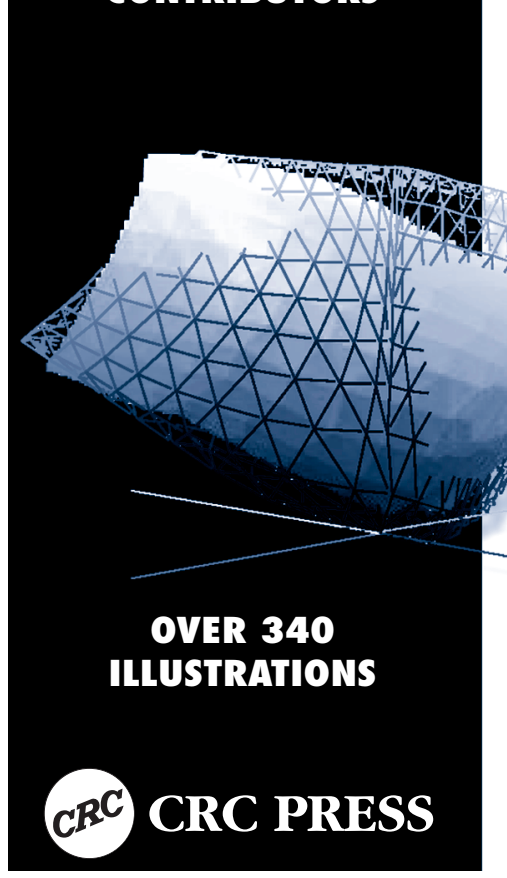
image compression, color quantization, gamut mapping, computatic
form algorithms, and color image processing for digital cameras.

Each chapter is written by world-class experts and largely self-contained, but cross references between chapters reflect the topics' important interrelations. Supplemental materials are available for download from the CRC Web site, including electronic versions of some of the images presented in the book.

\section{fectures}

Provides a single, comprehensive source of information on the science and technology of color imaging

- Begins with fundamentals and connects to state-of-the-art research in all areas of color imaging systems

- Emphasizes connections between chapters to facilitate the understanding of digital color imaging from a systems perspective-increasingly important in today's interconnected world

Covers recent innovations in the field of color imaging and image processing

- Includes extensive bibliographic references

$\checkmark$ Offers supplementary materials on the Web at www.crcpress.com 
Color Fundamentals for Digital Imaging, Gaurav Sharma, Xerox Corporation

Visual Psychophysics and Color Appearance, Garrett M. Johnson and Mark D. Fairchild, Rochester Institute of Technology

Physical Models for Color Prediction, Patrick Emmel, Clariant International

Color Management for Digital Imaging Systems, Edward J. Giorgianni, Thomas E. Madden and Kevin E. Spaulding, Eastman Kodak Company

Device Characterization, Raja Bala, Xerox Corporation
Digital Color Halftones, Charles $M$ Hains, Shen-ge Wang, and Keith T. Knox,

Xerox Corporation

Human Visual Model Based Color Halftoning, A. Ufuk Agar, Hewlett Packard Company, Farhan A. Baqai, Sony Electronics, and Jan P. Allebach, Purdue University

Compression of Color Images, Ricardo de Queiroz, Xerox Corporation

Color Quantization, Luc Brun, Université Reims, Champagne Ardenne and Alain Trémeau, Université Jean Monnet de Saint-Etienne
Gamut Mapping, Ján Morovič, University of Derby

Efficient Color Transformation Implementation, Raja Bala and $R$. Victor Klassen, Xerox Corporation

Color Image Processing for Digital Cameras, Ken Parulski and Kevin E. Spaulding, Eastman Kodak Company
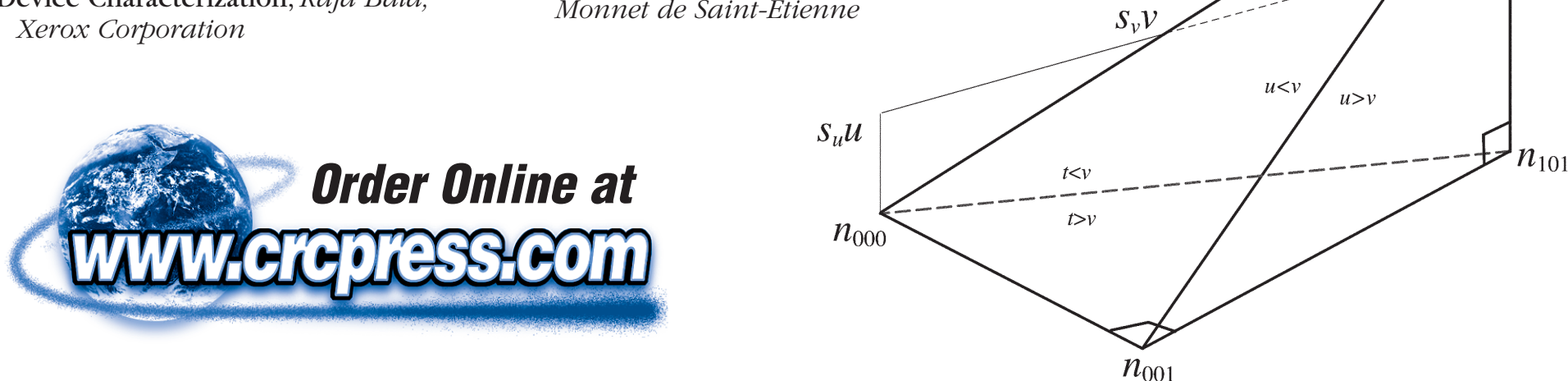

\section{Please use this ORDER FORM, CALL or ORDER ONLINE at WWW.CRCPRESS.COM}

Please indicate quantities next to the title(s) ordered below:

\section{DIGITAL COLOR IMAGING HANDBOOK}

..........Catalog no. 0900, ISBN: 0-8493-0900-X at \$129.95 / £87.00 each.

Other titles of interest:

\section{ADAPTIVE IMAGE PROCESSING: A COMPUTATIONAL}

INTELLIGENCE PERSPECTIVE

Catalog no. 0283, ISBN: 0-8493-0283-8 at \$109.95 / £72.99 each.

THE IMAGE PROCESSING HANDBOOK, FOURTH EDITION Catalog no. 1142, ISBN: 0-8493-1142-X at \$139.95/ £93.00 each.

THE DIGITAL SIGNAL PROCESSING HANDBOOK

Catalog no. 8572, ISBN: 0-8493-8572-5 at \$159.95 / £107.00 each.

Name .

$$
\text { please print clearly }
$$

Company/Institution

Address

City State/Province Zip/Postal Code
Ordering Information: Orders must be prepaid or accompanied by a purchase order. Checks should be made payable to CRC Press. Please add the appropriate shipping and handling charge for each book ordered. All prices are subject to change without notice. U.S./Canada: All orders must be paid in U.S. dollars. TAX: As required by law, please add applicable state and local taxes on all merchandise delivered to CA, FL, GA, IL, MA, NJ, NY, and Washington, DC. For Canadian orders, please add GST. We will add tax on all credit card orders. European Orders: All orders must be paid in U.K. £. VAT will be added at the rate applicable. Textbooks: Special prices for course adopted textbooks may be available for certain titles. To review a book for class adoption, contact our Academic Sales Department or submit your textbook evaluation request online at www.crcpress.com/eval.htm Satisfaction Guaranteed: If the book supplied does not meet your expectations, it may be returned to us in a saleable condition within 30 days of receipt for a full refund.

\begin{tabular}{|c|c|c|c|c|}
\hline \multicolumn{5}{|c|}{ SHIPPING AND HANDLING } \\
\hline Region & Delivery Time & First Title & Additional Title & \multirow{5}{*}{$\begin{array}{c}\text { For priority } \\
\text { mail services, } \\
\text { please contact } \\
\text { your nearest } \\
\text { CRC PRESS } \\
\text { office. }\end{array}$} \\
\hline USA/Canada & 3-5 Days & $\$ 5.99$ & $\$ 1.99$ & \\
\hline America/Asia/Australia & 7-14 Days & $\$ 9.99$ & $\$ 3.99$ & \\
\hline Europe & 3-5 Days & $£ 2.99$ & $£ 0.99$ & \\
\hline Middle East/Africa & 7-21 Days & $£ 4.99$ & $£ 2.99$ & \\
\hline
\end{tabular}

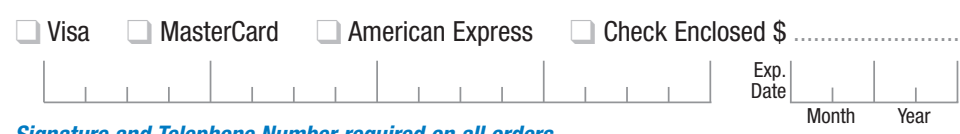

Signature and Telephone Number required on all orders .PO\#

Signature

Telephone

If you would like to receive information from us by e-mail, please provide your e-mail address below. E-Mail Address

\section{ORDERING LOCATIONS}

In North \& South America, Asia, and Australasia:

CRC PRESS

2000 N.W. Corporate Blvd. Boca Raton, FL 33431-9868, USA

Tel: 1-800-272-7737 • Fax: 1-800-374-3401

From Outside the Continental U.S.

Tel: 1-561-994-0555 • Fax: 1-561-361-6018 e-mail:orders@crcpress.com
In Europe, Middle East, and Africa:

CRC PRESS / ITPS Cheriton House, North Way Andover, Hants, SP10 5BE, UK Tel: 44 (0) 1264342932 Fax: 44 (0) 1264342788 e-mail: crcpress@itps.co.uk

\section{Corporate Offices}

CRC PRESS

2000 N.W. Corporate Blvd. Boca Raton, FL 33431-9868, USA

Tel: 1-800-272-7737 • Fax: 1-800-374-3401 From Outside the Continental U.S.

Tel: 1-561-994-0555 • Fax: 1-561-361-6018 e-mail:orders@crcpress.com
CRC PRESS UK

23-25 Blades Court, Deodar Road London SW15 2NU, UK

Tel: 44 (0) 2088754370

Fax: 44 (0) 2088713443

e-mail: enquiries@crcpress.com 